\title{
Severidade da desordem temporomandibular e sua relação com medidas cefalométricas craniocervicais
}

\author{
Severity of the temporomandibular disorder and its \\ relationship with craniocervical cephalometric measures
}

\author{
Jovana de Moura Milanesi ${ }^{[a]}$, Priscila Weber ${ }^{[\mathrm{b}]}$, Fernanda Pasinato ${ }^{[\mathrm{a}]}$, \\ Eliane Castilhos Rodrigues Corrêa ${ }^{[c]}$
}

[a] Fisioterapeutas, doutorandas do Programa de Pós-Graduação em Distúrbios da Comunicação Humana da Universidade Federal de Santa Maria (UFSM), Santa Maria, RS - Brasil, e-mails: jovanamil@yahoo.com.br, fepas.fisio@yahoo.com.br

[b] Fisioterapeuta, mestra em Distúrbios da Comunicação Humana pela Universidade Federal de Santa Maria (UFSM), Santa Maria, RS - Brasil, e-mail: prifisio07@yahoo.com.br

[c] Fisioterapeuta, professora adjunta do Departamento de Fisioterapia e Reabilitação e do Programa de Pós-Graduação em Distúrbios da Comunicação Humana da Universidade Federal de Santa Maria (UFSM), Santa Maria, RS - Brasil, e-mail: eliftrs@yahoo.com.br

\section{Resumo}

Introdução: Sabe-se que existe uma relação entre a Desordem Temporomandibular (DTM) e a postura craniocervical, porém, além de os estudos apresentarem resultados inconclusivos, a severidade dos sinais e sintomas de DTM não é considerada. Objetivo: Correlacionar índices de severidade da DTM com medidas cefalométricas craniocervicais. Materiais e métodos: Participaram da pesquisa 32 mulheres entre 19 e 35 anos com diagnóstico de DTM (RDC/TMD). A severidade dos sinais e sintomas da DTM foi avaliada pelo Índice Temoromandibular proposto por Pehling, calculado com base nos achados do exame físico do RDC/TMD (eixo I). A postura craniocervical foi avaliada por cefalometria, por meio de 11 medidas referentes à posição da cabeça, coluna cervical, mandíbula e osso hioide. A normalidade dos dados foi testada pelo teste de Lilliefors e as correlações foram realizadas pelo coeficiente de Spearman. Resultados: Foram encontradas correlações negativas e moderadas entre o ângulo CVT/Hor e os Índices Muscular ( $\mathrm{p}=0,0288$ ) e Temporomandibular $(\mathrm{p}=0,0394)$; entre o ângulo CPL/Hor (anteriorização) quando correlacionado aos Índices Funcional ( $p=0,0482)$ e Muscular $(p=0,0086)$ e entre distância do hioide à terceira vértebra cervical $(\mathrm{Hy} / \mathrm{C} 3)$ e o Índice Funcional $(\mathrm{p}=0,0155)$. Conclusões: Constatou-se associação entre a maior severidade do quadro clínico da DTM e a projeção anterior da cabeça, a flexão 
da coluna cervical baixa e a menor distância do osso hioide à terceira vértebra cervical. Essa relação sugere que as alterações posturais craniocervicais podem contribuir para a maior intensidade dos sinais e sintomas e perpetuação da DTM.

Palavras-chave: Transtornos da articulação temporomandibular. Postura. Cefalometria.

\section{Abstract}

Introduction: It is known that there is a relationship between the Temporomandibular Disorder (TMD) and craniocervical posture, but, besides the studies showed inconclusive results, the severity of signs and symptoms of TMD is not considered. Objective: To correlate the severity of TMD index to craniocervical cephalometric measurements. Materials and methods: Thirty-two women, from 19 to 35 years old with a diagnosis of TMD (RDC/TMD), took part in the study. The severity of signs and symptoms of TMD was assessed by the Temoromandibular Index proposed by Pehling and calculated according to the physical examination findings of the RDC/TMD (Axis I). The craniocervical posture was evaluated by cephalometry through 11 measures related to the head, cervical spine, jaw and the hyoid bone position. Data normality was tested by Lilliefors test and correlations were performed by Spearman coefficient. Results: Moderate negative correlation was found between the CVT/Hor angle and Muscular ( $p=0.0288)$ and Temporomandibular ( $p=0.0394)$ Indices; between the CPL/Hor angle (anterior) when correlated with Functional ( $p=0.0482)$ and Muscle indices $(p=0.0086)$ and between distance from the hyoid to the third cervical vertebra (Hy/C3) and Functional Index $(p=0.0155)$. Conclusions: There was an association between greater severity of clinical symptoms of TMD and a more forward head posture, the lower cervical spine flexion and a smaller distance from hyoid bone to the third cervical vertebra. This relationship suggests that the craniocervical postural changes may contribute to a greater intensity of signs and symptoms and the perpetuation of TMD.

Keywords: Temporomandibular joint disorders. Posture. Cephalometry.

\section{Introdução}

A Desordem Temporomandibular (DTM) é uma patologia do sistema estomatognático relativamente comum, que afeta cerca de 7 a $15 \%$ da população adulta, sendo mais frequente nas mulheres em idade produtiva (1). Caracteriza-se por sinais e sintomas como dor na articulação temporomandibular e nos músculos mastigatórios, limitação dos movimentos mandibulares e sons articulares (2).

Diversos questionários, índices e critérios diagnósticos têm sido utilizados na avaliação das DTMs. Chaves et al. (3) caracterizam o critério diagnóstico em pesquisa para DTM (RDC/TMD) como uma das mais bem estruturadas ferramentas para o diagnóstico da DTM. Entretanto, esse instrumento não qualifica a severidade da disfunção. 0 Índice Anamnésico de Fonseca é o instrumento mais utilizado para avaliar a severidade da DTM, embora algumas de suas questões não apresentem consistência adequada (4). Pehling et al. (5) propõem um índice para a classificação da severidade da DTM nos níveis funcional, muscular e articular abrangendo uma pontuação de 0 (ausência) a 1 (maior severidade).

A relação entre as DTMs e o sistema craniocervical vem sendo amplamente investigada, porém ainda sem evidências conclusivas. Estudos cefalométricos sustentam que a posição da cabeça e da coluna cervical interfere na postura da mandíbula e do osso hioide, e essas alterações podem estar associadas à dor craniomandibular $(6,7,8)$. Enfatizando essa relação entre coluna cervical e DTM, Von Piekartz e Lüdtke (9) encontraram um percentual importante de indivíduos com DTM em portadores de cefaleia cervicogênica e constataram uma redução significativa na sintomatologia cervical após tratamento específico da DTM, mesmo em longo prazo. Entretanto, outros estudos não relataram relações consistentes entre a postura craniocervical e a DTM $(10,11,12)$. Os autores acrescentam, ainda, que alterações na postura de cabeça e cervical são anormalidades encontradas igualmente em indivíduos com e sem DTM.

Com o intuito de contribuir para a elucidação dessas questões, este estudo propõe-se a verificar a 
severidade da DTM por meio dos índices funcional, muscular, articular e temporomandibular propostos por Pehling et al. (5) e sua relação com medidas cefalométricas craniocervicais.

\section{Materiais e métodos}

As participantes da pesquisa foram selecionadas dentre 94 mulheres que responderam voluntariamente mediante mídia impressa. Trinta e duas corresponderam aos critérios de inclusão e exclusão estabelecidos.

Foram incluídas mulheres com idade entre 19 e 35 anos e diagnóstico de DTM, conforme critério de diagnóstico para pesquisa em DTM - RDC/TMD (2). Os seguintes critérios de exclusão foram considerados: história de trauma facial e/ou craniocervical, presença de dor musculoesquelética aguda e intensa, deformidades musculoesqueléticas (escolioses, má formação óssea congênita), procedimentos cirúrgicos ortopédicos craniomandibulares e/ou cervicais e diagnóstico prévio de hérnia discal cervical, classificação de oclusão II e III de Angle, falhas dentárias, alterações oclusais do tipo mordida aberta anterior e posterior, mordida cruzada, mordida de topo e sobremordida, bem como uso de aparelho ortodôntico.

A pesquisa foi aprovada pelo Comitê de Ética em Pesquisa em Saúde da UFSM (n. 048.0.243.000-08) e as voluntárias foram informadas sobre os objetivos e procedimentos da pesquisa mediante o aceite e a assinatura do Termo de Consentimento Livre e Esclarecido.

\section{Diagnóstico da DTM}

0 exame físico do protocolo RDC/TMD (Eixo I) foi conduzido por um único examinador treinado (2). Esse instrumento classifica a DTM em subgrupos diagnósticos: desordens musculares (Grupo I), deslocamentos de disco (Grupo II) e artralgia, osteoartrite e osteoartrose (Grupo III). Um indivíduo pode receber no máximo um diagnóstico muscular (Grupo I), um diagnóstico do Grupo II e um diagnóstico do Grupo III para cada articulação, separadamente.

O Índice Temporomandibular (5) foi calculado com base nos achados clínicos do protocolo RDC/ TMD. Esse índice avalia a severidade da DTM abrangendo três domínios com atribuição de valores de
0 (ausência de sinal clínico) a 1 (presença de sinal clínico): índice funcional (mobilidade), índice muscular e índice articular. Para o índice funcional, são considerados 12 itens referentes aos movimentos mandibulares: abertura não assistida e assistida com e sem dor, lateralizações direita e esquerda, protrusão e padrão de abertura mandibular. 0 índice muscular contempla a palpação de 20 sítios referentes aos músculos mastigatórios extraorais temporais em seus três feixes, masseter em três regiões, região mandibular posterior, região submandibular e intraoralmente na área do pterigoide lateral e tendão do músculo temporal. E, finalmente, o índice articular, composto de oito itens considerando a palpação da articulação temporomandibular (polo lateral e ligamento posterior) e percepção de ruídos articulares como estalido nos movimentos de abertura, fechamento e lateralizações mandibulares e presença de creptações grosseiras e finas. 0 índice temporomandibular é composto pela média aritmética dos três índices, sendo considerada uma maior severidade dos sinais e sintomas de DTM quanto mais próximos de 1 forem os índices.

\section{Avaliação cefalométrica}

Os indivíduos foram submetidos a uma radiografia lateral direita do crânio e coluna cervical em posição ortostática habitual, ortostática adotando-se a posição natural da cabeça (PNC). Para reproduzir a $\mathrm{PNC}$, os indivíduos mantiveram o olhar fixo para o reflexo dos seus olhos no espelho localizado a $1 \mathrm{~m}$ de distância $(7,13,14)$.

As radiografias foram realizadas com o equipamento Orthophos Plus (Siemens, Alemanha), com o chassi posicionado paralelo à face e imediatamente adjacente ao ombro do indivíduo, para garantir a visualização de C7. Foi utilizado chassi com filme T MAT G Kodak de 18 x 24 cm, estando a distância foco-filme fixa em 1,52 m e em conformidade com o protocolo de radioproteção de acordo com a Portaria n. 453 de 01/06/1998 (Ministério da Saúde, Secretaria de Vigilância Sanitária).

Todos os ângulos foram traçados manualmente por um mesmo examinador previamente treinado. Os cefalogramas foram desenhados em papel acetato com auxílio de uma lapiseira (grafite $0,3 \mathrm{~mm}$ ) nas radiografias dispostas sobre um negatoscópio para melhor visualização das estruturas. Para as medidas 
angulares, adotou-se um transferidor, e para as medidas lineares, uma régua milimetrada. Com um intervalo de uma semana, 20 radiografias foram escolhidas aleatoriamente para uma segunda análise, a fim de verificar a confiabilidade do traçado realizado.

As variáveis analisadas no registro radiográfico lateral estão demonstradas na Figura 1. Foram calculadas 11 medidas, sendo sete referentes à posição da cabeça e coluna cervical e quatro referentes à posição mandibular e do osso hioide. As medidas craniocervicais avaliadas foram: NSL/OPT ou inclinação da base do crânio em relação à cervical superior; NSL/CVT ou inclinação da base do crânio em relação à cervical (C2 - C4); OPT/Hor ou inclinação da coluna cervical alta em relação à horizontal; CVT/Hor ou inclinação da coluna cervical baixa em relação à horizontal; EVT/CVT ou lordose cervical; CVA ou flexoextensão da cabeça; e CPL/Hor ou anteriorização da cabeça.
Quanto às medidas mandibulares e do osso hioide, foram analisados o ângulo NSL/ML ou inclinação da base do crânio em relação à mandíbula, Hy/ML ou distância do hioide ao plano mandibular, Hy/C3 ou distância do hioide à terceira vértebra cervical e Hy/ Me ou distância do hioide ao mento.

\section{Análise estatística}

A normalidade dos dados foi testada pelo teste de Lilliefors. A correlação entre os índices funcional (mobilidade), muscular, articular e temporomandibular com as variáveis referentes à postura craniocervical e de posição mandibular e hioidea medidas pela cefalometria foi realizada pelo coeficiente de Spearman. Este varia de -1 (correlação negativa, variáveis seguem em sentido contrário) a 1 (correlação positiva,

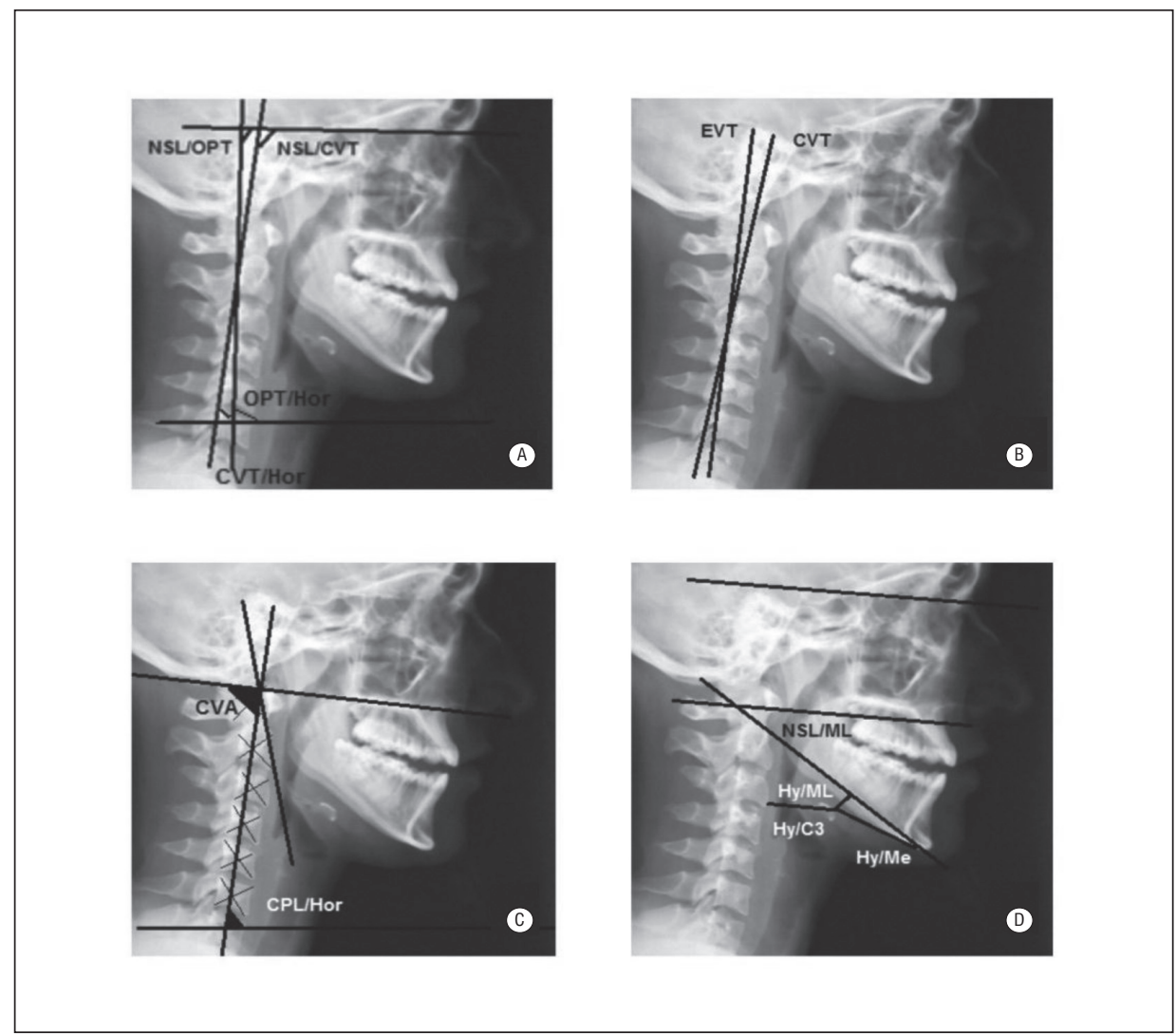

Figura 1 - Variáveis cefalométricas craniocervicais e de posição mandibular e hioidea

Legenda: A) NSL/OPT = Inclinação da base do crânio em relação à cervical superior; NSL/CVT = Inclinação da base do crânio em relação à cervical (C2-C4); OPT/Hor = Inclinação da coluna cervical alta em relação à horizontal; CVT/Hor = Inclinação da coluna cervical baixa em relação à horizontal; B) EVT/CVT = Lordose cervical; C) CVA = Flexoextensão da cabeça; CPL/Hor = Anteriorização; D) NSL/ML = Inclinação da base do crânio em relação à mandíbula; $\mathrm{Hy} / \mathrm{ML}=$ Distância do hioide ao plano mandibular; Hy/C3 = Distância do hioide à terceira vértebra cervical; Hy/Me = Distância do hioide ao mento.

Fonte: Dados da pesquisa. 
variáveis seguem no mesmo sentido). A correlação foi considerada forte para valores de coeficiente de correlação (r) $\geq 0,7$; moderada quando $0,3<\mathrm{r}<0,7$, e fraca quando $0<\mathrm{r}<0,3$ (15). Considerou-se um nível de significância de $5 \%$.

\section{Resultados}

Com relação aos diagnósticos de desordem temporomandibular, $87,5 \%$ das participantes $(\mathrm{n}=28)$ foram diagnosticadas com DTM miofascial, 46,9\% $(\mathrm{n}=15)$ apresentaram DTM mista (presença de dor miofascial associada a pelo menos um diagnóstico do Grupo II e/ou III) e $12,5 \%(n=4)$ receberam diagnóstico de DTM articular (Grupo III).

No Quadro 1 estão expressos os resultados referentes à correlação entre as variáveis cefalométricas craniocervicais e os índices de severidade de DTM. Uma correlação negativa e moderada foi encontrada entre CVT/Hor e os índices muscular ( $p=0,0288$ ) e temporomandibular $(p=0,0394)$, ou seja, menores valores angulares (maior inclinação anterior da coluna cervical baixa) corresponderam à maior severidade nesses dois índices. Da mesma forma comportou-se o ângulo CPL/Hor (anteriorização), quando correlacionado aos índices funcional $(\mathrm{p}=0,0482) \mathrm{e}$ muscular $(p=0,0086)$. Assim, quanto maior a posição de anteriorização da cabeça, maior a severidade dos sinais e sintomas relacionados aos movimentos mandibulares e músculos mastigatórios.

Os valores referentes às correlações entre as variáveis cefalométricas mandibulares e hioideas e a severidade dos sinais e sintomas de DTM encontram-se no Quadro 2. Apenas uma correlação negativa moderada foi observada entre a distância do hioide à terceira vértebra cervical (Hy/C3) e o índice funcional $(p=0,0155)$, sugerindo que uma menor distância pode estar associada a um maior prejuízo nos movimentos mandibulares.

\section{Discussão}

Sabe-se que posição da cabeça interfere diretamente na postura da mandíbula, o que explica a existência de uma forte comorbidade entre DTM e cervicalgia, sugerindo que elas podem compartilhar fatores de risco ou até influenciar uma a outra (6, $16,17)$. Além disso, há evidências de uma forte relação entre a incapacidade mandibular e da coluna cervical (18).

Considerando a presença de uma possível associação entre a dor craniomandibular com alterações posturais no segmento craniocervical, a cefalometria tem sido utilizada como ferramenta de análise do

Quadro 1 - Correlação entre as variáveis cefalométricas craniocervicais e os índices funcional, muscular, articular e temporomandibular

\begin{tabular}{ccccc}
\hline Variáveis & IF & IM & IA & IT \\
\hline NSL/OPT & 0,2194 & 0,2533 & $-0,0662$ & 0,1348 \\
NSL/CVT & 0,1820 & 0,2766 & $-0,0378$ & 0,1662 \\
OPT/Hor & $-0,1940$ & $-0,1038$ & $-0,0965$ & $-0,1881$ \\
CVT/Hor & $-0,2482$ & $-\mathbf{0 , 3 8 6 5 ^ { * }}$ & $-0,1350$ & $-\mathbf{0 , 3 6 6 1 ^ { * }}$ \\
EVT/CVT & 0,0208 & $-0,0324$ & $-0,0776$ & $-0,0334$ \\
CVA & $-0,1508$ & $-0,1300$ & $-0,2128$ & $-0,2822$ \\
CPL/Hor & $-\mathbf{0 , 3 5 2 0 ^ { * }}$ & $\mathbf{- 0 , 4 5 6 4 ^ { * }}$ & 0,1513 & $-0,2602$ \\
\hline
\end{tabular}

Legenda: IF = Índice funcional; IM = Índice muscular; IA = Índice articular; IT = Índice temporomandibular; NSL/OPT = Inclinação da base do crânio em relação à cervical superior; NSL/CVT = Inclinação da base do crânio em relação à cervical (C2 - C4); OPT/Hor = Inclinação da coluna cervical alta em relação à horizontal; CVT/Hor = Inclinação da coluna cervical baixa em relação à horizontal; EVT/CVT = Lordose cervical; CVA = Flexoextensão da cabeça; CPL/Hor $=$ Anteriorização. Nível de significância: * $=p<0,05$

Fonte: Dados da pesquisa.

Nota: Resultados expressos em valor de r (coeficiente de correlação de Spearman). 
Quadro 2 - Correlação entre as variáveis cefalométricas mandibulares e hioideas e os índices funcional, muscular, articular e temporomandibular

\begin{tabular}{|c|c|c|c|c|}
\hline Variáveis & IF & IM & IA & IT \\
\hline NSL/ML & $-0,0584$ & 0,3460 & $-0,1265$ & $-0,0238$ \\
\hline $\mathrm{HY} / \mathrm{ML}$ & $-0,1421$ & 0,0791 & 0,0121 & $-0,1031$ \\
\hline HY/C3 & $-0,4241^{*}$ & $-0,2444$ & 0,1912 & $-0,1925$ \\
\hline HY/ME & $-0,0378$ & 0,0505 & 0,1662 & 0,1345 \\
\hline
\end{tabular}

Legenda: IF = Índice funcional; IM: Índice muscular; IA = Índice articular; IT = Índice temporomandibular; NSL/ML = Inclinação da base do crânio em relação à mandíbula; $\mathrm{Hy} / \mathrm{ML}=$ Distância do hioide ao plano mandibular; Hy/C3 = Distância do hioide à terceira vértebra cervical; $\mathrm{Hy} / \mathrm{Me}=$ Distância do hioide ao mento. Nível de significância: * $=p<0,05$.

Fonte: Dados da pesquisa.

Nota: Resultados expressos em valor de r (coeficiente de correlação de Spearman).

alinhamento da cabeça e da coluna cervical em indivíduos com e sem DTM $(7,8)$. Sendo assim, a tecnologia na análise de imagem deve ser usada para proporcionar informações fidedignas e auxiliar os profissionais nas decisões referentes à postura cervical e sua associação com dor e disfunção (19).

As correlações negativas e moderadas encontradas entre o ângulo CVT/Hor e os índices muscular e temporomandibular nesta pesquisa sugerem a relação entre uma maior inclinação cervical baixa e uma maior severidade da DTM, considerando todos os componentes do índice temporomandibular. A flexão da coluna cervical baixa é uma alteração biomecânica componente da anteriorização de cabeça (20). Nessa posição, há um desequilíbrio entre os músculos extensores e flexores da cabeça, os músculos mastigatórios e os músculos supra e infra-hioideos $(21,22)$, que podem interferir nas funções do sistema estomatognático.

Na presença de uma postura de cabeça anteriorizada, ocorre uma rotação posterior do crânio e a mandíbula tende a se retrair, levando o côndilo mandibular a uma posição mais posterior (6). Tal hipótese foi confirmada em um estudo com 15 adultos saudáveis, mostrando que a postura anteriorizada da cabeça proporciona uma posição significativamente mais posterior do côndilo mandibular quando comparada à postura natural da cabeça (22). Alguns estudos têm sugerido que o posicionamento condilar posterior pode estar associado a sinais e sintomas de DTM, especialmente ao deslocamento de disco $(23,24)$.

Neste estudo, foram encontradas correlações negativas moderadas entre a variável CPL/Hor (anteriorização) e os índices funcional e muscular, indicando que quanto maior a posição de anteriorização da cabeça, maior a severidade dos sinais e sintomas relacionados aos movimentos mandibulares e músculos mastigatórios.

Armijo-Olivo et al. (21) utilizaram a telerradiografia para avaliar a postura da cabeça e cervical de 25 indivíduos com DTM comparados a 25 controles saudáveis. Observaram uma tendência nos indivíduos com deslocamento anterior do disco de apresentarem rotação posterior da cabeça, diminuição do espaço funcional C0-C1 e diminuição da lordose cervical. Apesar disso, Andrade et al. (8) investigaram alterações estruturais da cabeça e do pescoço de 17 indivíduos com DTM e 17 controles por meio de radiografias e não encontraram diferenças significativas entre os grupos. Arimijo-Olivo et al. (12) destacam que pequenas diferenças posturais encontradas entre indivíduos com e sem DTM podem não ter relevância sob o ponto de vista clínico.

Em outro estudo, Weber et al. (25) observaram relação entre a presença de DTM e o grau de severidade da disfunção cervical, porém atribuíram esse achado à inervação comum do complexo trigêmino-cervical e à hiperalgesia de indivíduos com DTM e não a alterações posturais craniocervicais. Convém destacar que nenhum desses estudos associou a severidade dos sinais e sintomas da DTM às alterações da postura craniocervical.

Com relação às medidas mandibulares e hioideas, apenas a variável referente à distância horizontal entre o hioide e a terceira vértebra cervical (Hy/C3) apresentou uma correlação negativa e moderada com o índice funcional. A adoção de uma postura 
anteriorizada da cabeça pode resultar no aumento da tensão dos músculos supra e infra-hioideos, por isso a mandíbula é tracionada para trás e para baixo, fazendo com que a articulação temporomandibular assuma uma postura viciosa (26). Assim, prejuízos funcionais podem ocorrer. Além disso, maior flexão da coluna cervical baixa pode também ser responsável pela diminuição da distância Hy/C3, levando à aproximação entre o osso hioide e a coluna cervical.

0 instrumento critério diagnóstico em pesquisa para DTM (RDC/TMD) é considerado uma das melhores ferramentas para o diagnóstico da DTM (3). Entretanto, por meio dele não é possível conhecer o grau de severidade da disfunção. Felício et al. (1) destacam a importância do uso de questionários com escalas numéricas para severidade combinadas aos instrumentos diagnósticos de DTM. Nesse sentido, o índice temporomandibular proposto por Pehling et al. (5) avalia a severidade da DTM quantitativamente, embora não permita a classificação do grau de severidade.

Bevilaqua-Grossi et al. (27), avaliando sinais e sintomas cervicais e a severidade da DTM de 100 mulheres com disfunção, constataram que os sinais e sintomas na coluna cervical acompanham a severidade da DTM, mas que o inverso não é verdadeiro. Assim, quanto maior a severidade da DTM, maior a severidade da disfunção cervical; porém uma maior disfunção cervical não determina uma maior severidade de DTM.

Em um estudo comparando a atividade elétrica dos músculos cervicais de indivíduos com e sem DTM, Milanesi et al. (28) observaram maior atividade elétrica no grupo DTM, salientando a relação entre a coluna cervical e a DTM. Os autores recomendam a realização de estudos com uma faixa etária mais elevada e a avaliação da severidade da DTM. Dessa forma, além da avaliação da presença ou não da disfunção, destaca-se a importância da investigação da severidade da DTM, bem como das alterações na postura de cabeça e pescoço.

\section{Conclusão}

Foram observadas correlações moderadas entre medidas cefalométricas indicativas de anteriorização da cabeça e aproximação entre o osso hioide à terceira vértebra cervical com índices de severidade da DTM. Embora os resultados não permitam estabelecer uma relação de causa e efeito entre estas variáveis, essa associação sugere que as alterações posturais craniocervicais podem contribuir para a maior intensidade dos sinais e sintomas e a perpetuação da DTM. A importância clínica desse achado reafirma a importância de avaliar e reabilitar aspectos posturais relacionados ao equilíbrio entre o sistema estomatognático e craniocervical.

\section{Referências}

1. Felício CM, Ferreira CLP, Medeiros APM, Silva MAMR, Tartaglia GM, Sforza C. Electromyographic indices, orofacial myofunctional status and temporomandibular disorders severity: a correlation study. J Electromyogr Kinesiol. 2012;22(2):266-72. doi:10.1016/j.jelekin.2011.11.013. doi:10.1016/j. jelekin.2011.11.013.

2. Dworkin SF, Leresche L. Research diagnostic criteria for temporomandibular disorders: review, criteria, examinations and specifications, critique. J Craniomandib Disord. 1992;6(4):301-55. PMid:1298767.

3. Chaves TC, Oliveira AS, Bevilaqua-Grossi D. Principais instrumentos para avaliação da disfunção temporomandibular, parte II: critérios diagnósticos; uma contribuição para a prática clínica e de pesquisa. Rev Fisioter Pesq. 2008;15(1):101-6.

4. Campos JADB, Gonçalves DAG, Camparis CM, Speciali JG. Reliability of a questionnaire for diagnosing the severity of temporomandibular disorder. Rev Bras Fisioter. 2009;13(1):38-43. doi:10.1590/ S1413-35552009005000007.

5. Pehling J, Schiffman E, Look J, Shaefer J, Lento P, Fricton J. Interexaminer reliability and clinical validity of the temporomandibular index: a new outcome measure for temporomandibular disorders. J Orofac Pain. 2002;16(4):296-304. PMid:12455430.

6. Rocabado M. Biomechanical relatonship of cranial, cervical and hyoid regions. J Craniomandibular Pract. 1983;1(3):61-6. PMid:6586872.

7. Visscher CM, De Boer W, Lobbezzo F, Habets LL, Naeije M. Is there a relationship between head posture and craniomandibular pain? J Oral Rehabil. 2002;29(11):1030-6. doi: 10.1046/j.1365-2842.2002.00998.x.

8. Andrade AV, Gomes PF, Salmela TLF. Cervical spine alynment and hyoid bone positioning with temporomandibular disorders. J Oral Rehabil. 2007;34(10):767-72. doi:10.1111/j.1365-2842.2006.01698.x. 
9. Von Piekartz H, Lüdtke K. Effect of Treatment of Temporomandibular Disorders (TMD) in patients with cervicogenic headache: a single-blind, randomized controlled study. Cranio 2011;29(1):43-56. PMid:21370769.

10. Iunes DH, Carvalho LCF, Oliveira AS, Bevilaqua-Grossi D. Análise da postura cranio-cervical em pacientes com disfunção temporomandibular. Rev. Bras. Fisioter. 2009;13(1):89-95. doi:10.1590/ S1413-35552009005000011.

11. Matheus RA, Ramos-Perez FM, Menezes AV, Ambrosano GM, Haiter-Neto F, Bóscolo FN, et al. The relationship between temporomandibular dysfunction and head and cervical posture. J Appl Oral Sci. 2009;17(3):2048. doi:10.1590/S1678-77572009000300014.

12. Armijo-Olivo S, Rappaport K, Fuentes J, Gadotti IC, Major PW, Warren S, et al. Head and cervical posture in patients with temporomandibular disorders. J Orofac Pain. 2011;25(3):199-209. PMid:21837287.

13. Solow B, Sonnesen L. Head posture and malocclusion. Eur J Orthod. 1998;20(6):685-93. doi:10.1093/ ejo/20.6.685.

14. Rosa LP, Moraes LC. Estudo comparativo da influência do método de posicionamento convencional e natural de cabeça para obtenção de radiografias laterais cefalométricas utilizando análise crânio-cervical. Cienc Odontol Bras. 2009;12(1):56-62.

15. Chan VH. Biostatistics 104: correlational Analysis. Singapore Med. J. 2003;44(12):614-619. PMid:14770254.

16. Cuccia A, Caradonna C. The relationship between the stomatognathic system and body posture. Clinics. 2009;64(1):61-6. doi:10.1590/S180759322009000100011.

17. Wiesinger B, Malker H, Englund E, Wänman A. Does a dose-response relation exist between spinal pain and temporomandibular disorders? BMC Musculoskelet Disord. 2009;10(28):1-8.

18. Olivo SA, Fuentes J, Major PW, Warren S, Thie NMR, Magee DJ. The association between neck disability and jaw disability. J Oral Rehabil. 2010;37(9):670-9. doi:10.1111/j.1365-2842.2010.02098.x.

19. Grimmer-Somers K, Milanese S, Louw Q. Measurement of cervical posture in sagittal plane. J Manipulative Physiol Ther. 2008;31(7):509-17. doi:10.1016/j. jmpt.2008.08.005.
20. Neiva PD, Kirkwood RN, Godinho R. Orientation and position of head posture, scapula and thoracic spine in mouth-breathing children. Int J Pediatr Otorhinolaryngol. 2009;73(2):227-36. doi:10.1016/j. ijporl.2008.10.006.

21. Armijo-Olivo S, Silvestre R, Fuentes J, da Costa BR, Gadotti IC, Warrenet S, et al. Clinic and teleradiographic alterations in pacients with anterior disc displacement with reduction. Kinesiologia 2001;64:82-7.

22. Omure H, Miyawaki S, Nagata J, Ikeda K, Yamasaki K, Alkalaly A, et al. Influence of forward head posture on condilar position. J Oral Rehabil. 2008;35(11):795800. doi:10.1111/j.1365-2842.2007.01834.x.

23. Cholausueska P, Warita H, Soma K. Alterations of the rat temporomandibular joint in functional posterior displacement of the mandible. Angle Orthod. 2004; 74(5):677-83.

24. Di Paolo C, D'Ambrosio F, Panti F, Papa M, Marcini P. The condyle-fossa relationship in temporomandibular disorders. Minerva Stomatol. 2006;55(7-8):409-22. PMid:17041541.

25. Weber P, Corrêa ECR, Ferreira FS, Soares JC, Bolzan GP, Silva AM. Cervical spine dysfunction signs and symptoms in individuals with temporomandibular disorder. J Soc Bras Fonoaudiol. 2012;24(2):134-9. doi:10.1590/S2179-64912012000200008.

26. Biasotto-Gonzalez DA. Abordagem interdisciplinar das disfunções temporomandibulares. São Paulo: Manole; 2005.

27. Bevilaqua-Grossi D, Chaves TC, de Oliveira AS. Cervical spine signs and symptoms: perpetuating rather than predisposing factors for temporomandibular disorders in women. J Appl Oral Sci. 2007;15(4):259-264. doi:10.1590/S1678-77572007000400004.

28. Milanesi JM, Corrêa ECR, Borin GS, Souza JA, Pasinato F. Avaliação eletromiográfica e músculos mastigatórios com uso de acupuntura em indivíduos com desordem temporomandibular. Rev Fisioter Pesq. 2011;18(3):217-22

Recebido: 27/06/2012 Received: 06/27/2012

Aprovado: 09/12/2012 Approved: 12/09/2012 